\title{
Evaluation of method in systematic reviews and meta-analyses published in LIS
}

\author{
Kalyani Ankem
}

\begin{abstract}
The objective of the research was to evaluate systematic reviews and metaanalyses appearing in library and information science (LIS) literature. LIS databases were searched to find systematic reviews and meta-analyses published during 1996 - 2006 in LIS journals. Seven systematic reviews and five metaanalyses were selected and methods applied (identification of studies, inclusion/exclusion criteria, quality assessment, data extraction, and synthesis) were reviewed. The evaluation indicates that all systematic reviews in LIS have been published on medical library or medical information topics. Searching conducted to identify studies for systematic reviews and meta-analyses is comprehensive. Inclusion/exclusion criteria and quality standards established in systematic reviews and meta-analyses are well developed; however, in most systematic reviews, the analysis is limited to percentages rather than inferential statistics. Analysis based on inferential statistics in systematic reviews will further improve the reviews. The meta-analyses published in LIS illustrate different approaches to conducting the synthesis.
\end{abstract}

\section{Introduction}

Systematic reviews provide a research method for conducting literature reviews according to a set of procedures - systematically - and for synthesizing existing results on a research problem or a research question (Carr, 2002). Consequently, a systematic review allows the researcher to review and assess knowledge in important areas. Such a review of knowledge can be applied effectively to improve practice; thus, a systematic review also facilitates evidence-based practice. In evidence-based practice, systematic reviews provide a method for creating the often missing link between research and practice. Ultimately, a systematic review serves to inform by drawing from a body of literature and summarizing the results. When an investigator also mathematically combines the results from existing findings, the method applied is referred to as meta-analysis (Lipsey and Wilson, 2000).

\section{Kalyani Ankem}

Dr. Kalyani Ankem is an associate professor in the School of Library and Information Sciences at North Carolina Central University. She teaches research methods, health information resources, and online searching.

\section{E-mail: kankem@,nccu.edu}

Received January 2008 
Although systematic reviews and meta-analyses have been applied widely in disciplines such as medicine, nursing, psychology, and business, library and information science (LIS) has been relatively slow to adopt the method despite its value. This article aims to review systematic reviews and meta-analyses published in LIS and report on the status of application of the methodology in the field.

\section{Method}

To review the status of systematic reviews and meta-analyses in LIS, the LISA and Library Literature databases were searched to retrieve publications representative of systematic reviews and meta-analyses conducted by authors in LIS. The focus was on contributions made by authors in LIS; that is, authors affiliated with libraries, information centers, or LIS schools listed on LIS studies Libraryschools, universities, and departments (Danish Royal School of Library and Information Science Library, 2007).

A search for publications with the terms systematic review, meta-analysis, or meta-analytic appearing in the title was conducted in both LISA and Library Literature databases. This search for articles which included the term(s) in the title was conducted to retrieve only those publications in which a systematic review or meta-analysis was the main focus. A traditional literature review was not considered a systematic review. Both database searches in LISA and Library Literature were limited to years $1996-2006$. The reasoning behind limiting the search to a ten-year period from $1996-2006$ was to analyze systematic reviews and meta-analysis published more recently, thereby examining the most developed applications of the methodology with an intent to report on the status of the application of the methodology in LIS.

In LISA, 46 citations were found, and in Library Literature, 23 citations were retrieved. All citations retrieved were for articles appearing in journals. This article is limited to the evaluation of method in quantitative systematic reviews and meta-analyses. To select quantitative systematic reviews and meta-analyses published by authors in LIS, the following criteria were applied to the citations retrieved:

- The title of the journal is listed on BUBL: Library and Information Science Journals (BUBL Information Service, 2007) or The Mortimore-Singh Guide to Publication in Library and Information Studies (University of North Carolina at Greensboro, 2007), or the term(s) library, libraries, or librarianship appear in the title of the journal;

- The primary author of the article is affiliated with a library, information center, or an LIS school listed on LIS studies - Libraryschools, universities, and departments (Danish Royal School of Library and Information Science Library, 2007);

- The article is published in English;

- The article is a quantitative systematic review as described by Carr (2002) or an application of a meta-analytic technique as described by Lipsey and Wilson (2000) or Lyons (2003); 
- The article is an application of the method and not a discussion of the method.

It is important to emphasize that only quantitative reviews (systematic reviews or meta-analyses) were to be covered for the evaluation. The following is a brief description of journals and articles removed from the pool selected.

From the citations retrieved in LISA, 22 articles published in the following journals were eliminated because they were not listed as an LIS journal in $B U B L$ : Library and Information Science Journals (BUBL Information Service, 2007) or The Mortimore-Singh Guide to Publication in Library and Information Studies (University of North Carolina at Greensboro, 2007).

1. British Medical Journal (two articles)

2. Document Design (one article)

3. European Journal of Communication (one article)

4. Human-Computer Interaction (one article)

5. International Journal of Human-Computer Studies (two articles)

6. Journal of Communication (two articles)

7. Journal of Database Management (one article)

8. Journal of Educational Multimedia and Hypermedia (one article)

9. Journal of Information Technology (one article)

10. Journal of Medical Internet Research (five articles)

11. Journal of Organizational and End User Computing (one article)

12. Journal of End User Computing (one article)

13. Learning Organization (one article)

14. Methods of Information in Medicine (one article)

15. Scientometrics (one article)

Additionally, three articles were eliminated from the citations retrieved in LISA because the primary author was not affiliated with a library, information center, or an LIS school. The journals in which the articles appeared were the following:

1. Information and Software Technology (primary author affiliated with Simula Research Laboratory, Lysaker, Norway).

2. Journal of Scholarly Publishing (primary author affiliated with Department of Educational Leadership and Policy Studies, Iowa State University, Ames, IA, USA).

3. Information Resources Management Journal (primary author affiliated with Business Information Systems, Central Michigan University, Mount Pleasant, MI, USA).

A further nine articles were removed from the citations retrieved in LISA because they did not meet one or more of the other criteria listed earlier. 
All citations retrieved in Library Literature constituted articles published in journals listed in either BUBL: Library and Information Science Journals (BUBL Information Service, 2007) or The Mortimore-Singh Guide to Publication in Library and Information Studies (University of North Carolina at Greensboro, 2007) or in journals including the term(s) library, libraries, or librarianship in the title. However, several citations (14) retrieved in Library Literature were for articles about the methodology rather than for those that were an application of the methodology. Thus, these were eliminated from the pool. Also eliminated from the citations retrieved in Library Literature were an article appearing in the Journal of Scholarly Publishing (primary author was not affiliated with a library, information center, or an LIS school), an article which included the term metaanalysis in the title but was not a meta-analysis as described by Lipsey and Wilson (2000) or Lyons (2003), and an article in a journal published in Chinese. The citations for these three articles were retrieved in LISA as well.

The final pool of selected articles consisted of seven systematic reviews and five meta-analyses. These articles attempt a synthesis of the literature and were published in LIS journals. Also, the primary author of the selected articles was affiliated with a library, information center, or an LIS academic entity.

This process of sample selection is illustrated in Figure 1. 


\section{LISA:}

Retrieved 46 articles.

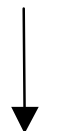

Excluded 22 articles published in journals that were not listed on BUBL: Library and Information Science Journals or The Mortimore-Singh Guide to Publication in Library and Information Studies.

Excluded 3 articles in which the primary author was not affiliated with a library, information center, or an LIS school.

Excluded 9 articles that did not meet the inclusion criteria established for the evaluation.

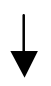

7 systematic reviews and 5 meta-analyses met inclusion criteria for the evaluation.

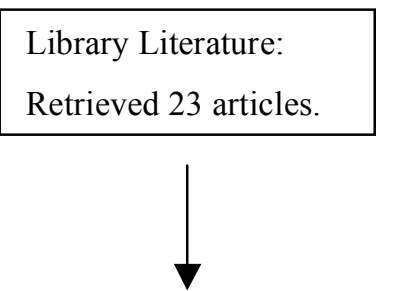

Excluded 14 articles that discussed the method and were not an application of the method.
Excluded 3 articles that did not meet the inclusion criteria established for the evaluation

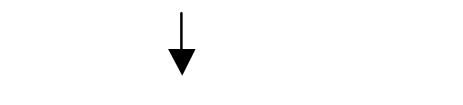

2 systematic reviews and 4 meta-analyses met inclusion criteria for the evaluation

Figure 1: Selection of systematic reviews and meta-analyses for the evaluation from LISA and Library Literature databases 


\section{Systematic reviews and meta-analyses published in LIS during 1996- 2006}

Interestingly, all the retrieved systematic reviews conducted in LIS were on medical library or medical information topics. The questions probed in these systematic reviews fall mainly in three areas: clinical librarianship, information skills training, and patient information needs.

Three of the seven systematic reviews probe the effectiveness of clinical librarianship (CL). CL involves the provision of "quality-filtered information to health professionals at the point of need to support clinical decision-making" (Winning and Beverley, 2003, 10). Winning and Beverley's review, published in 2003, builds upon Cimpl's (1985) work in the eighties and attempts to provide evidence for the effectiveness of CL services. Winning and Beverley studied whether CL services were used by clinicians and whether the services affect patient care and/ or clinicians' use of literature in practice. Evidence indicated that clinicians used or planned to continue to use CL services.

During the following two years, Wagner and Byrd (2004) and Weightman and Williamson (2005) published two reviews in the same area. Wagner and Byrd undertook a substantial study to review literature from thirty years on the programs of clinical medical libraries (CML) to provide evidence for their effectiveness. The authors studied the various impacts, including patient care impact, of clinical librarian programs. The analysis indicated that firstly, the perceived usefulness and quality and secondly, improved patient care are the strongest impacts of CML services. Around the same time, Weightman and Williamson conducted a review of literature specifically to find the value and impact of library services on health outcomes for patients. Their analysis separates the impact of clinical librarian services from that of traditional librarian services. Studies suggested impacts from traditional librarian services on general patient care, diagnosis, choice of tests, choice of therapy, and reduced length of stay. Impacts from clinical librarian services included those on diagnosis, choice of drugs/therapy, and treatment/management.

Two systematic reviews focused on topics related to producing effective information skills training in the health field. Brettle conducted a systematic review to identify effective methods of information skills training and to determine whether information skills training improves search skills as well as affects patient care (Brettle, 2003). According to the author, however, there was "limited evidence for the effect of training on skills, insufficient evidence for most effective training methods, and limited evidence for the effect of training on patient care" (ibid, 3). Childs et al.'s (2005) systematic review of the literature, part of an in-house project, was carried out to understand the barriers to, and solutions for, e-learning in the process of online training in the health field. The authors present a listing of barriers and solutions gathered from the studies that they included.

Ankem published two systematic reviews in 2005 and 2006 (Ankem, 2005b; Ankem, 2006b). Both were on the information needs of cancer patients. The first review sought to determine the types of information needs of cancer patients and 
any influencing factors (Ankem, 2005b). A synthesis showed that information about the disease itself and information about the treatment that follows are the most important types. A review of factors affecting the need for a particular type of information found that

1) younger patients attribute more importance to information about sexual concerns and physical attractiveness than older patients, and

2) patients who preferred to be active in decision-making during illness want more information about their illness.

The objectives of the second systematic review were to determine cancer patients' use of information sources and to identify the factors that influence this use (Ankem, 2006b). Health care professionals, medical pamphlets, and family and friends were the most used information sources. Books, health care professionals and medical pamphlets were found to be the most helpful information sources. Also, younger patients used health care professionals and certain forms of written information sources more than older patients.

Meta-analysis has been rarely conducted in LIS. However, the topics on which meta-analysis has been conducted are varied. In the early nineties, Trahan tested the potential for meta-analysis in LIS via a pilot study on paper versus computerbased retrieval (Trahan, 1993). The present article in its evaluation covers metaanalyses published during 1996 - 2006, and it was not until 1996 that a study that was described as a meta-analysis was published. In 1996, Salang published a meta-analysis on the relationship between user information needs and information retrieval in an unknown journal (Salang, 1996). The perusal of the study failed to convey clear intent and/ or logic in the choice of variables examined for the analysis. Published in 1997, Haug's meta-analysis on physician's preferences for information sources, which were ranked, can be considered a precursor to metaanalysis in LIS as it lacks the synthesis of existing results found in other metaanalyses (Haug, 1997). That is, no mathematical synthesis is performed in Haug's study.

Therefore, this author considers Saxton's meta-analysis, also published in 1997, as the first substantive contribution to meta-analysis in LIS (Saxton, 1997). Saxton's meta-analysis compares observed correlations between reference accuracy and other variables. Total expenditures, number of volumes added, fluctuation in collection, size of the service population, and weekly hours of operation at the library showed a moderate association with reference accuracy.

Ankem's meta-analysis, published in 2006, probed the influence of various factors on the level of cancer patients' information needs (Ankem, 2006a). The pooled effect sizes that were produced showed that younger cancer patients and patients who preferred active roles in treatment decision-making need more information. As did two systematic reviews discussed above, the most recent meta-analysis focused on information skills training. Published a few months after Ankem's (2006a) meta-analysis, Koufogiannakis and Wiebe's (2006) meta-analysis sought to find which library instruction method was most effective for improving the information skills of students at an introductory, undergraduate level. The analysis suggested that traditional instruction is more effective than no instruction. 


\section{Evaluation of method: findings}

In LIS, more systematic reviews (seven) exist than meta-analyses (five). The following is a review of approaches to systematic reviews and meta-analyses used by the authors in LIS.

\subsection{Identification of studies (searching)}

The strength of the present systematic reviews, all of which are on medical library or medical information topics, is searching. All of the reviews, especially the more recent, include elaborate search strategies which hold as models to researchers interested in conducting systematic reviews, even those researchers who are outside LIS. These comprehensive strategies for searching are a contribution of LIS to the method. This contribution is significant because comprehensive searching is critical to systematic reviews. Particularly in metaanalysis, an incomplete search means that omitted results can possibly overturn the outcome or results of the meta-analysis.

For guidance on comprehensive searching, the reader is advised to refer to Brettle (2003) and Weightman and Williamson (2005), in particular. Listings of keywords and subject headings and any strategies or limits used in searching the selected databases are explained in detail, often in several steps. Ankem (2006a) and Koufogiannakis and Wiebe (2006) in their meta-analyses also undertake and present elaborate searching. Koufogiannakis and Wiebe (2006), in addition, provide an extremely structured search process in the appendix to their article. Koufogiannakis and Wiebe's search strategies stand as a model for other researchers to follow.

\subsection{Inclusion or exclusion criteria}

Inclusion or exclusion criteria are established to select studies based on types of studies, types of participants, types of interventions (if relevant), and most importantly, types of outcome measures (Winning and Beverley, 2003).

Several systematic reviews include both qualitative and quantitative studies. For instance, while Childs and colleagues provide an extensive list of inclusion or exclusion criteria, all types of research designs and even various discussions of issues including expert viewpoints were considered for inclusion in their review (Childs et al, 2005). Brettle (2003) and Wagner and Byrd (2004) also include quantitative and qualitative studies.

This synthesis of data from quantitative and qualitative studies in the same set of results in a systematic review can be likened to the analogy of combining apples and oranges. The idea behind systematic reviews is to synthesize similar data across studies in one set of results. That is, while qualitative and quantitative results may be reported in the same review, it is advised that these data not be synthesized in the same set of results. It is possible that the authors of systematic reviews were utilizing all types of data available in the same set of results for practical purposes. That is, some of the issues encountered in applying the established inclusion or exclusion criteria can be attributed to the absence of sufficient evidence in the literature for analysis. 
Especially in a meta-analysis, heterogeneous results when mathematically combined become statistically meaningless. Therefore, authors are required to present justification for the selection of studies chosen before mathematically combining data in a meta-analysis. Consequently, a more rigorous application of criteria is undertaken in the meta-analyses. The meta-analyses in LIS indicate that the inclusion or exclusion criteria have been applied rigorously. Saxton (1997), Ankem (2006a), and Koufogiannakis and Wiebe (2006) discuss the application of the criteria in the selection of individual studies in great detail.

\subsection{Quality assessment}

The process of evaluating studies based on quality is often referred to as critical appraisal. The systematic reviews provide considerable guidance on setting quality standards. Quality standards are established to evaluate studies, most importantly, those based on reliability, validity, and applicability (Winning and Beverley, 2003).

Wagner and Byrd (2004) followed Friedman, Owens, and Wyatt's (2001) criteria for medical informatics evaluative studies and further appraised the papers in their review using additional criteria including adequacy of sample, relevance of hypotheses, and reliability. Winning and Beverley applied the Critical Appraisal Checklist developed by CriSTAL: Critical Skills Training in Appraisal for Librarians, which includes validity, reliability, and applicability standards (Winning and Beverley, 2003). Brettle evaluated studies using an instrument developed by the Health Care Practice R\&D Unit (HCPRDU) (Brettle, 2003). This tool covers quantitative, qualitative and multi-method studies. Weightman and Williamson appraised the papers in their review using internationally accepted criteria that appeared in previously published literature (Weightman and Williamson, 2005). Among meta-analyses, only Koufogiannakis and Wiebe (2006) used a structured checklist based on previous approach.

As noted above, the quality standards established in the reviews provide guidance for others to follow. However, the authors were unable to apply the standards rigorously. After establishing quality standards, Winning and Beverley did not exclude studies based on quality (Winning and Beverley, 2003). Weightman and Williamson (2005) offer a pragmatic approach by providing an alternative in those instances where it is difficult to apply quality standards due to insufficient data. They adjusted their standards to include only those studies that met a certain response rate and, at least, two (not all) of their quality criteria (Weightman and Williamson, 2005). The authors of meta-analyses faced similar issues.

\subsection{Data extraction}

Once the inclusion or exclusion criteria and quality standards are established and individual studies are selected, the methodology stage of the systematic review process involves the extraction of data for synthesis. As such, the conduct of systematic reviews or meta-analyses is heavily dependent on quantity and quality of existing results. In LIS, almost all of the authors of systematic reviews and meta-analyses agree concerning the lack of existing results available for synthesis in the literature. According to the authors, the evidence that exists is insufficient 
and/ or generally of poor quality (Brettle, 2003; Childs et al, 2005; Wagner and Byrd, 2004; Weightman and Williamson, 2005; Winning and Beverley, 2003).

Another observation made by authors of systematic reviews and meta-analyses is the scatter in terms of research questions analyzed (Childs et al, 2005; Wagner and Byrd, 2004). A wide range of research questions are analyzed across the literature on a topic, and the body of literature is not a cohesive whole, thus complicating and perhaps disallowing the gathering and synthesis of results. To emphasize this insufficiency, Wagner and Byrd note the difficulty they faced in extracting results to pull together common themes (Wagner and Byrd, 2004). Even the outcome measures or variables within studies are often not operationalized or not described adequately to allow other researchers to understand the exact nature of the variable(s) under study for inclusion in the systematic review. For instance, traditional librarian services and clinical librarian services analyzed in Weightman and Williamson's review are not clearly defined in the studies that the authors include (Weightman and Williamson, 2005). Weightman and Williamson attempt to clarify the definitions of these services in their review.

Information on the reliability and validity of methods used in studies that authors wish to include in systematic reviews is not present or available (Winning and Beverley, 2003). Often, sample sizes and the necessary statistical parameters associated with the question of interest are missing from the studies selected for analysis. As a practical approach, for instance, Wagner and Byrd estimate the missing numbers of users or uses of clinical medical library service from the studies to arrive at weighted averages that represent different positive user evaluations of clinical medical library service (Wagner and Byrd, 2004).

The authors of meta-analyses also point to similar issues in finding evidence for synthesis - limited evidence, lack of quality, inadequate reporting of methods and statistics, and scatter in research questions. However, as these authors could mathematically combine the results, they were able to find more evidence, at least, adequate for a meta-analysis (Ankem, 2006a; Koufogiannakis and Wiebe, 2006; Saxton, 1997).

\subsection{Synthesis}

The systematic reviews have evolved over time. In earlier systematic reviews, the results lack true synthesis; also, not enough information is presented in the results (Brettle, 2003). In Childs et al. (2005), the synthesis of data concerning barriers and solutions to effective e-learning for health professionals and students is a listing of findings gathered across studies. Winning and Beverley (2003) did not yet present their results on the effectiveness of clinical librarianship in elaborate tables; however, they did provide the results with statistics (percentages), although few. Some of their results even included Likert scale values, but it is not clear whether the Likert scale values represent mean or median values. Sufficient details on statistics when included in the review would assist readers in understanding the data included in synthesis. More elaborate presentations of results appeared in Weightman and Williamson (2005), Wagner and Byrd (2004), and Ankem (2005b; 2006b). Weightman and Williamson (2005) and Wagner and 
Byrd (2004) presented their results on the effectiveness of clinical librarianship in an extremely systematic fashion with copious tables. The synthesis, however, is limited to percentages extracted from studies. Again, a lack of inferential statistics in existing studies may have forced this limitation.

All three meta-analyses (Ankem, 2006a; Koufogiannakis and Wiebe, 2006; Saxton, 1997) provided elaborate analysis. Saxton (1997) and Ankem (2006a), in their meta-analyses, synthesized correlations found across studies. As noted earlier, Saxton analyzed the association between multiple variables and reference accuracy, and Ankem analyzed the association between demographic and situational variables and the level of information needs of cancer patients (Ankem, 2006a). Koufogiannakis and Wiebe applied a different meta-analytic approach and synthesized mean differences found across studies to find the effectiveness of various instructional methods in developing information literacy skills

(Koufogiannakis and Wiebe, 2006). The authors of the three meta-analyses faced several of the issues encountered by the authors of systematic reviews, although perhaps to a lesser extent; they were able to analyze the literature comprehensively, find inferential statistics representative of an association between variables of interest to them, and synthesize the findings. Also, the different approaches to meta-analyses serve as excellent examples for others in the LIS field who may be interested in applying the meta-analytic method.

Two suggestions for synthesis in future meta-analyses to improve further the method include the following. Saxton limited analysis to correlations found in studies (Saxton, 1997), but researchers are encouraged to use all inferential statistics representative of associations of interest because they can be converted into correlations. Saxton's approach was conservative; given the paucity of data, the latter approach is recommended. Saxton's updated recommendations for meta-analyses found in an article published in Library Trends include this suggestion (Saxton, 2006).

Also, Koufogiannakis and Wiebe, in their meta-analysis, enter several pairs of comparisons found within one study between firstly traditional instruction and new instruction and secondly self-directed learning and no instruction separately when combining mean differences found across studies (Koufogiannakis and Wiebe, 2006). This multiplicity may have the effect of inflating the results. The literature on meta-analysis advises that researchers average the results of multiple comparisons between the same variables from a single study before entering them for analysis to avoid inflating results. Also, when an individual study included comparison of one self-directed learning group with two no instruction groups, Koufogiannakis and Wiebe divided the self-directed learning group into two groups (ibid). This was achieved by halving the sample size and entering each of the no instruction groups separately in analysis. If the original finding in the preceding was a result of ANOVA, the mean differences from follow-up tests should have been used instead. 


\section{Conclusions}

The evaluation provides evidence of substantial contributions on systematic reviews to medical library and information literature. Several advances have been made in developing the application of the methodology. Searching, inclusion or exclusion criteria and quality standards are well developed and provide guidance for others interested in conducting systematic reviews. However, the application of inclusion or exclusion criteria and quality standards and the analysis at a higher level using inferential statistics (parameters from t-tests, correlations, chi-square tests), rather than analysis limited to simple descriptive statistics such as percentages, require attention. The premise of systematic reviews is to gather similarly conducted studies. Therefore, qualitative results must be analyzed separately from quantitative results.

The existing meta-analyses have been approached more rigorously in terms of application of both inclusion or exclusion criteria and quality standards. Worthy of note is that these studies have been conducted in varied areas; that is, not all three meta-analyses focus on medical library or medical information topics.

In applying the method, researchers in LIS will find Saxton's article on the method (Saxton, 2006) and Ankem's (2005a) article on conducting meta-analysis to be helpful. These articles provide references for additional reading, especially seminal materials, in meta-analysis. The most rigorous meta-analyses can be conducted through collaborations between practitioners and researchers as ultimately a meta-analysis is designed to improve practice. Furthermore, statisticians should be consulted for quantitative reviews. In general, researchers must adequately and clearly define variables and report with all necessary parameters (sample sizes, test statistics, probabilities of significance), so rigorous meta-analyses can be conducted on important topics.

\section{References}

Ankem, K. (2005a) Approaches to meta-analysis: a guide for LIS researchers, Library \& Information Science Research 27 (2), 164-176.

Ankem, K. (2005b) Types of information needs among cancer patients: a systematic review, LIBRES 15 (2), URL:

http://libres.curtin.edu.au/libres15n2/index.htm [accessed 15.03.07].

Ankem, K. (2006a) Factors influencing information needs among cancer patients: a meta-analysis, Library \& Information Science Research 28 (1), 7-23.

Ankem, K. (2006b) Use of information sources among cancer patients: results of a systematic review of the research literature, Information Research 11(3), URL: http://informationr.net/ir/11-3/paper254.html [accessed 15.03.07].

Brettle, A. (2003) Information skills training: a systematic review of the literature, Health Information and Libraries Journal 20 (Suppl.1), 3-9.

BUBL Information Service (2007) BUBL: Library and Information Science Journals. URL: http://bubl.ac.uk/journals/bycat.htm [accessed 24.05.07].

Carr, A.B. (2002) Systematic reviews of the literature: The overview and metaanalysis, Dental Clinics of North America 46 (1), 79-86. 
Childs, S., et al (2005) Effective e-learning for health professionals and students barriers and their solutions: a systematic review of the literature - findings from the HeXL project, Health Information and Libraries Journal 22 (Suppl.2), 20-32.

Cimpl, K. (1985) Clinical medical librarianship: a review of the literature, Bulletin of the Medical Library Association 73 (1), 21-8.

Danish Royal School of Library and Information Science Library (2007) LIS studies - Libraryschools, universities, and departments. URL: http://www.db.dk/dbi/internet/schools.htm [accessed 20.05.07].

Friedman, C.P., Owens, D.K. and Wyatt, J.C. (2001) Evaluation and technology assessment. In: Shortliffe, E.H. and Perreault, L.E., eds. Medical informatics: computer applications in health care and biomedicine, New York, Springer, 282323. ISBN 0387984720.

Haug, J.D. (1997) Physicians' preferences for information sources: a meta-analytic study, Bulletin of the Medical Library Association 85 (3), 223-32.

Koufogiannakis, D. and Wiebe, N. (2006) Effective methods for teaching information literacy skills to undergraduate students: a systematic review and meta-analysis, Evidence Based Library and Information Practice 1 (3), URL: http://ejournals.library.ualberta.ca/index.php/eblip/issue/view/9 [accessed 15.03.07].

Lipsey, M.W. and Wilson, D.B. (2000) Practical meta-analysis: applied social research methods, London, Sage Publications. ISBN 0761921680.

Lyons, L.C. (2003) Meta-analysis: methods of accumulating results across research domains. URL: http://www.lyonsmorris.com/MetaA/index.htm [accessed 20.11.07].

Salang, M. M. C. (1996) A meta-analysis of studies on user information needs and their relationship to information retrieval, Journal of Philippine Librarianship 18 (2), 36-56.

Saxton, M.L. (1997) Reference service evaluation and meta-analysis: findings and methodological issues, Library Quarterly 67 (3), 267-89.

Saxton, M.L. (2006) Meta-analysis in library and information science: method, history, and recommendations for reporting research, Library Trends 55 (1), 15870.

Trahan, E. (1993) Applying meta-analysis to library and information science research, Library Quarterly 63 (1), 73-91.

University of North Carolina at Greensboro (2007) The Mortimore-Singh Guide to Publication in Library and Information Studies. URL:

http://www.uncg.edu/lis/Publication\%20Guide/pages/about this_guide.htm [accessed 20.05.07].

Wagner, K.C. and Byrd, G.D. (2004) Evaluating the effectiveness of clinical medical librarian programs: a systematic review of the literature, Journal of the Medical Library Association 92 (1), 14-33. 
Weightman, A.L. and Williamson, J. (2005) The value and impact of information provided through library services for patient care: a systematic review, Health Information and Libraries Journal 22 (1), 4-25.

Winning, M.A. and Beverley, C.A. (2003) Clinical librarianship: a systematic review of the literature, Health Information and Libraries Journal 20 (suppl.1), $10-21$ 